\title{
Ein Thymom als seltene Ursache extrakardialer Thoraxschmerzen
}

\author{
Thymoma as a Rare Cause of Non-Cardiac Chest Pain
}

Autoren

Institute
F. C. Ringshausen ${ }^{1}$, C. M. Heyer ${ }^{2}$, J. Reichert ${ }^{3}$, G. Rohde ${ }^{4}$

Die Institutsangaben sind am Ende des Beitrags gelistet. $\begin{array}{ll}\text { eingereicht } 23.1 .2012 & 2010\end{array}$ akzeptiert nach Revision 15. 2. 2012

\section{Bibliografie}

Dol http://dx.doi.org/ 10.1055/s-0031-1291875

Online-Publikation: 13.3 .2012

Pneumologie 2012; 66: 423-425

(c) Georg Thieme Verlag KG

Stuttgart · New York

ISSN 0934-8387

Korrespondenzadresse Dr. med. Felix C. Ringshausen Klinik für Pneumologie Medizinische Hochschule Hannover

Carl-Neuberg-Str. 1 30625 Hannover Ringshausen.Felix@mhhannover.de
Ein 70-jähriger Ex-Raucher (abstinent seit 30 Jahren, kumulativ 15 Packungsjahre) wurde vom Hausarzt zur Abklärung eines großen intrathorakalen Tumors vorgestellt ( $\bullet$ Abb.1).

Er gab an, seit zwei Jahren an einem belastungs-, atem- und bewegungsunabhängigen, durch Kompression nicht auslösbaren, nicht ausstrahlenden, leicht bis mäßig ausgeprägten, dumpf-drückenden, linksseitigen Thoraxschmerz zu leiden. Aufgrund der gleichen Beschwerden war genau ein Jahr zuvor eine (vermeintlich unauffällige) Koronarangiografie durchgeführt und die Diagnose „extrakardialer Thoraxschmerzen“ gestellt worden ( Abb. 2).

Eine vorgeschaltete Ergometrie, eine Echokardiografie und ein Röntgen-Thorax waren damals nicht durchgeführt worden. Die weitere Anamnese des Patienten war unauffällig. Bei der Auskultation des Thorax fiel ein pulssynchrones „Schwirren“ auf Höhe des fünften Interkostalraums in der linken vorderen Axiallarlinie auf. Die übrige körperliche Untersuchung sowie die laborchemische und apparatetechnische Basisdiagnostik einschließlich EKG, Echokardiografie, Lungenfunktion und flexibler Bronchoskopie ergaben keine wegweisenden Befunde. Die weiterführende Schnittbilddiagnostik konnte eine Infiltration der Brustwand und benachbarter Strukturen weitgehend ausschließen ( Abb.3). Gemäß interdisziplinärem Konsensus wurde eine diagnostische und therapeutische anterolaterale Thorakotomie mit makro- und mikroskopisch vollständiger Resektion des Tumors einschließlich seiner intakten und nicht infiltrierten Kapsel durchgeführt $(\bullet$ Abb.4). Auf eine radikale mediastinale Lymphadenektomie konnte daher verzichtet werden. Histologisch zeigte sich ein undifferenzierter, teils lymphatische, teils epitheliale Strukturen ausbildender Tumor ( $\bullet$ Abb.5). Mit Hilfe der ergänzenden Immunhistochemie gelang es schließlich, die definitive Diagnose eines im Gesunden entfernten, gemischtzelligen Thymoms vom histologi- schen Typ AB nach WHO-Klassifikation [1], Stadium I nach Masaoka-Stadieneinteilung [2], pT1cN0cM0 nach Yamakawa-Masaoka-TNMKlassifikation [3] zu stellen. Der weitere postoperative Verlauf gestaltete sich komplikationslos. Eine adjuvante Tumortherapie war nicht indiziert. Die regelmäßigen Kontrolluntersuchungen im Rahmen der Tumornachsorge verliefen im bisher vierten Jahr nach Resektion unauffällig. Primäre Tumore des Thymus sind mit einer Inzidenz von 0,15 pro 100000 insgesamt sehr selten. Sie machen bis zu 20\% aller Mediastinaltumoren aus und stellen die häufigsten Tumoren im vorderen Mediastinum dar. Thymome besitzen eine außerordentliche histologische Heterogenität und sind definiert als benigne, nicht maligne organoide Tumoren des Thymusepithels mit variabler, nicht neoplastischer, meist unreifer T-Zell-Komponente [4]. Sie können eine vielgestaltige klinische Präsentation und eine einzigartige Vielzahl an assoziierten paraneoplastischen autoimmunen Syndromen aufweisen [5], die bei unserem Patient jedoch nicht vorlagen. In ca. $40 \%$ finden sich lokale, durch Tumorkompression verursachte Symptome. Das Vorhandensein einer Myasthenia gravis scheint, möglicherweise aufgrund der frühzeitigeren Diagnosestellung, mit einer günstigeren Langzeitprognose assoziiert zu sein [6]. Als weitere prognostische Faktoren dient neben der Histologie das Ausmaß der möglichst kompletten chirurgischen Tumorentfernung.

Dieser Fall unterstreicht die Bedeutung der rationalen Stufendiagnostik von Thoraxschmerzen: anstelle der sofort durchgeführten Koronarangiografie, während der das Thymom in der Durchleuchtung zwar dargestellt, aber nicht bemerkt worden war, wäre die nicht-invasive Ursachenklärung durch eine sorgfältige Anamnese, eine Belastungsuntersuchung, eine gezielte Ultraschalldiagnostik und eine Röntgenaufnahme des Thorax indiziert und so bereits ein Jahr früher möglich gewesen. 


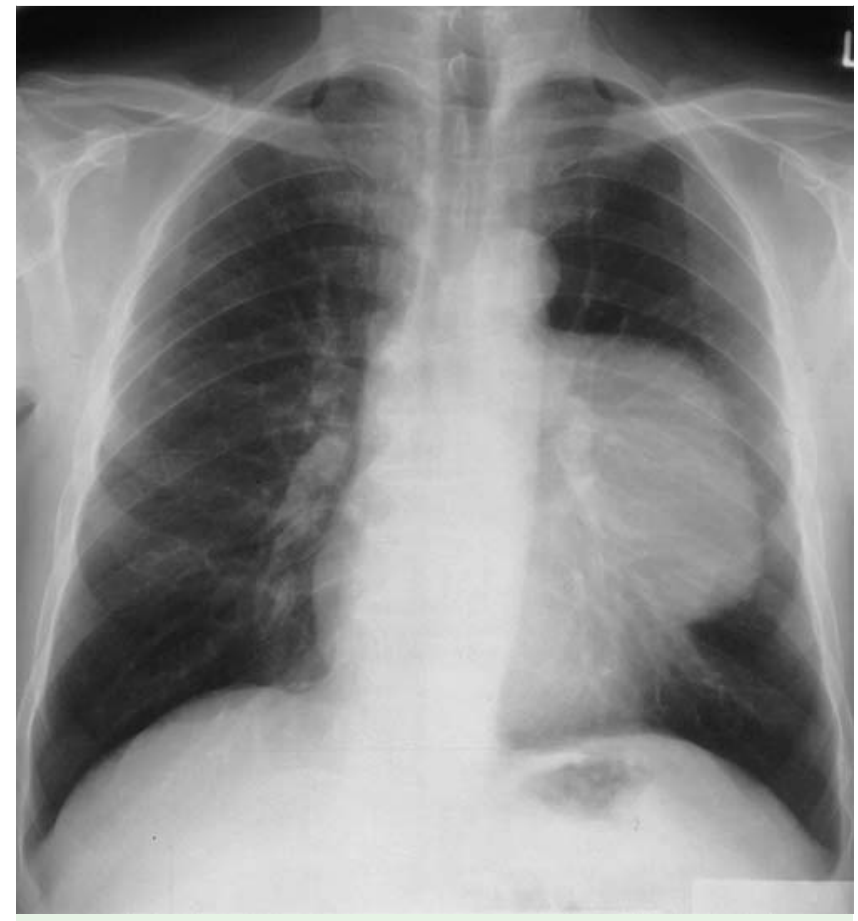

Abb. 1 Extern angefertigter Röntgen-Thorax in posterior-anteriorer Projektion: große links parakardial gelegene Raumforderung. Röntgen-Voraufnahmen existierten nicht.

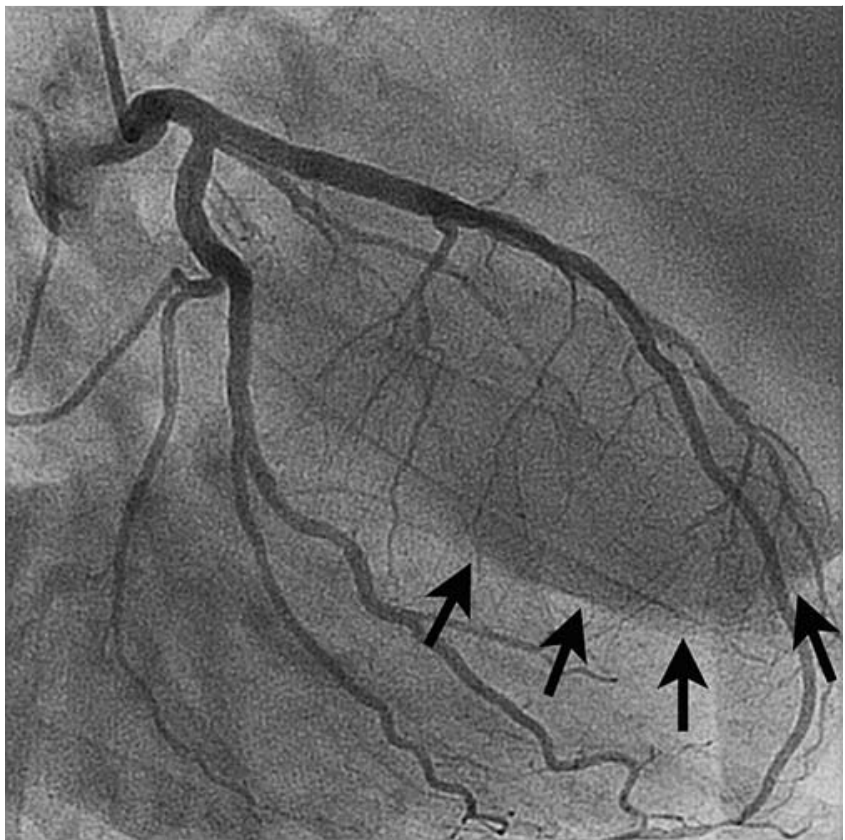

Abb.2 Kritische Durchsicht der ein Jahr zuvor durchgeführten Koronarangiografie (linke Koronararterie in rechtsanteriorer Schrägprojektion, RAO $30^{\circ}$ ): bereits damals sichtbare Kontur eines großen Tumors (Pfeile), sodass eine langsame Progredienz des Prozesses naheliegt.

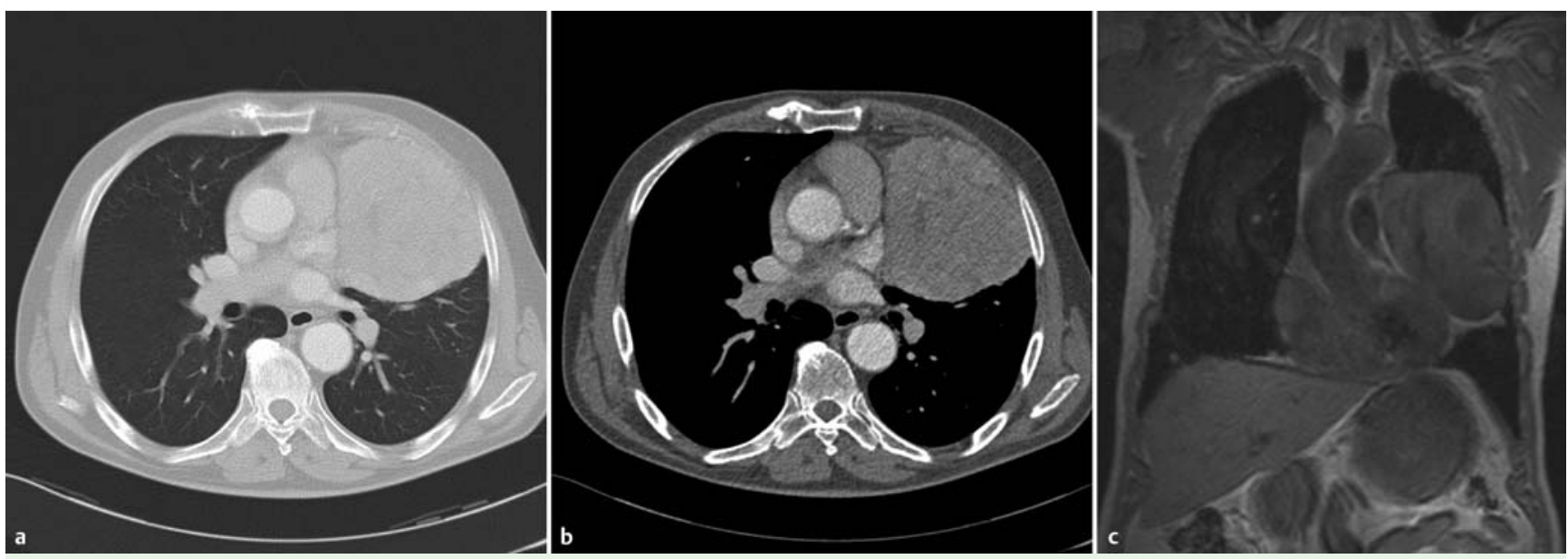

Abb.3 Axiale Rekonstruktion der kontrastmittelverstärkten Computertomografie des Thorax im Lungen- (a) und Weichteilfenster (b) und native T1-gewichtete, koronare Magnetresonanztomografie (c): $12 \times 8 \times 10 \mathrm{~cm}$ großer, paramediastinal und pleural gelegener, vom vorderen Mediastinum ausgehender, gut vaskularisierter und glatt begrenzter Tumor.
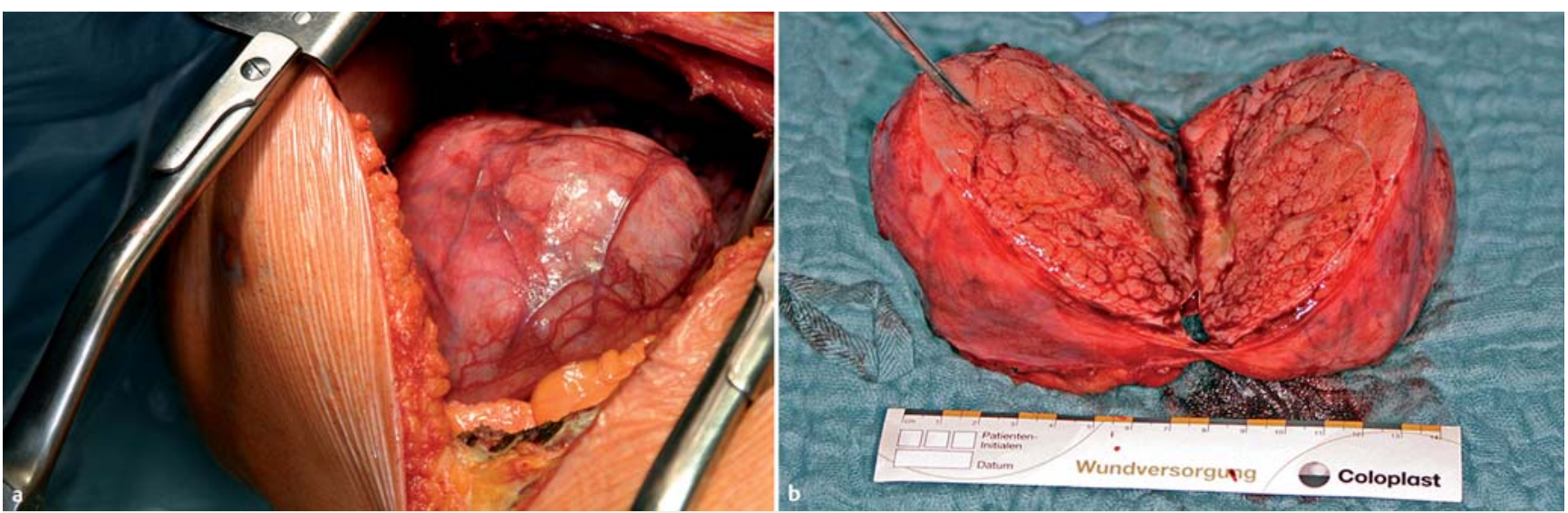

Abb.4 Intraoperativer Befund: intrapleural und extrapulmonal gelegener, mediastinal adhärenter, leicht ablösbarer, derber und gut vaskularisierter, ca. $530 \mathrm{~g}$ schwerer Tumor in situ (a), mit einer „fleischig“-gelappt imponierenden Schnittfläche und zarter, intakter Kapsel nach Resektion in toto (b). 

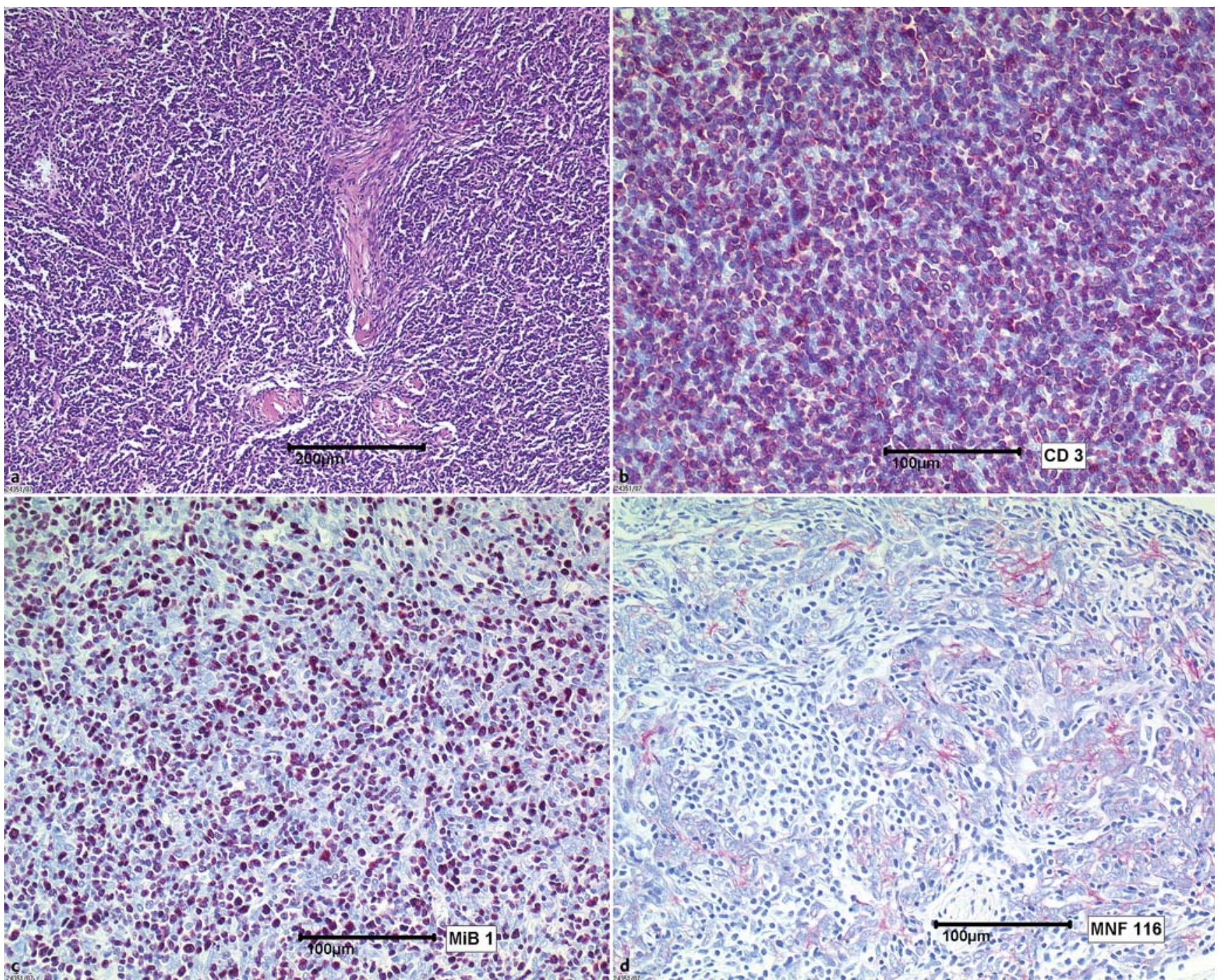

Abb.5 Histologischer und immunhistochemischer Befund: mäßig zellreicher Tumor, aus dicht gelagerten chromatindichten Zellen bestehend, die herdförmig z. T. in einem mukoiden Stroma gelagert sind (a, Hämatoxylin-Eosin-Färbung); deutlich positive Reaktion des überwiegenden Anteils der lymphatischen Zellen innerhalb des Tumors (b, T-Zell-Marker CD3); deutliche proliferative Aktivität der lymphozytären Komponente mit einem Proliferationsgrad von ca. $80 \%$ (c, Proliferationsmarker MiB-1); positive Reaktion der Zellkomplexe mit mäßiger Darstellung und Ausbildung netzartig anastomosierender Zellkomplexe (d, Keratin MNF116).

\section{Danksagung \\ $\nabla$}

Die Autoren danken Frau Prof. Dr. med. A. Tannapfel, Institut für Pathologie am Berufsgenossenschaftlichen Universitätsklinikum Bergmannsheil, Institut der Ruhr-Universität Bochum für das histologische Bildmaterial.

\section{Interessenkonflikt}

$\nabla$

Die Autoren geben an, dass kein Interessenkonflikt besteht.

Institute

${ }^{1}$ Klinik für Pneumologie, Medizinische Hochschule Hannover 2 Institut für Diagnostische Radiologie, Interventionelle Radiologie und Nuklearmedizin, Berufsgenossenschaftliches Universitätsklinikum Bergmannsheil, Klinikum der Ruhr-Universität Bochum ${ }^{3}$ Klinik für Herz- und Thoraxchirurgie, Berufsgenossenschaftliches Universitätsklinikum Bergmannsheil, Klinikum der Ruhr-Universität Bochum ${ }^{4}$ Department of Respiratory Medicine, Maastricht University Medical Centre, Maastricht, Niederlande

\section{Literatur}

1 Rosai J, Sobin LH. Histological typing of tumours of the thymus. WHO International histological classification of tumours. 2nd ed. Berlin-Heidelberg-New York: Springer; 1999

2 Masaoka A, Monden Y, Nakahara K et al. Follow-up study of thymomas with special reference to their clinical stages. Cancer 1981; 48: 2485 2492

3 Yamakawa Y, Masaoka A, Hashimoto T et al. A tentative tumor-nodemetastasis classification of thymoma. Cancer 1991; 68: 1984-1987

4 Tumours of the thymus. In: Travis WD, Brambilla E, Muller-Hermelink HK, Harris CC, editors. World Health Organization Classification of Tumours. Pathology and Genetics of Tumours of the Lung, Pleura, Thymus and Heart. 3 ed. Lyon: IARC Press; 2004

5 Muller-Hermelink HK, Marx A. Thymoma. Curr Opin Oncol 2000; 12: $426-433$

6 Margaritora S, Cesario A, Cusumano G et al. Thirty-five-year follow-up analysis of clinical and pathologic outcomes of thymoma surgery. Ann Thorac Surg 2010; 89: 245-252 discussion 252 